\title{
BOUNDARY INTEGRAL METHODS IN BIOELECTROMAGNETICS AND BIOMEDICAL APPLICATIONS OF ELECTROMAGNETIC FIELDS
}

\author{
DRAGAN POLJAK ${ }^{1}$, MARIO CVETKOVIĆ ${ }^{1}$, DAMIR CAVKA ${ }^{1}$, ANDRES PERATTA ${ }^{2}$, \\ CRISTINA PERATTA ${ }^{2}$, HRVOJE DODIG ${ }^{3} \&$ AKIMASA HIRATA $^{4}$ \\ ${ }^{1}$ University of Split, Split, Croatia \\ ${ }^{2}$ Wessex Institute of Technology, Southampton, UK \\ ${ }^{3}$ Marine Center for Electronics, Split, Croatia \\ ${ }^{4}$ Nagoya Institute of Tech., Japan
}

\begin{abstract}
The paper reviews some integral methods used in static, low frequency (LF), and high frequency (HF) dosimetry, respectively, and biomedical applications of electromagnetic fields. Laplace equation/Boundary element method (BEM) is applied to certain electrostatic and LF exposure scenarios. Surface Integral Equation (SIE)/Method of Moments (MoM) and tensor volume integral equation/MoM and Stratton Chu integral formulation coupled with vector Helmholtz equations/hybrid Boundary element method (BEM)/Finite element method (FEM) scheme are used to analyze the exposure to HF radiation. In addition to human exposure to undesired electromagnetic radiation sources, this work also deals with biomedical applications of electromagnetic fields, by using SIE/MoM approach. Some illustrative numerical results for induced current densities, electric field and SAR, are also given in the paper.
\end{abstract}

Keywords: human exposure to electromagnetic fields, integral formulation, numerical methods, Static, low and high frequency dosimetry, biomedical applications of electromagnetic fields.

\section{INTRODUCTION}

Human exposure to non-ionising radiation has caused public concern regarding possible adverse health effects [1]. The assessment of low frequency (LF) exposure involves the calculation of internal current density [2] and internal electric field [3], while high frequency (HF) exposure requires the calculation of specific absorption rate (SAR) and resultant temperature increase in a tissue. Measurement of internal fields is generally not possible and human exposure assessment is undertaken by means of sophisticated anatomically based computational models [4]-[7] mostly related to the use of the Finite Difference Time Domain (FDTD) methods. The Finite Element Method (FEM), Boundary Element Method (BEM), Method of Moments (MoM) and some other methods are used to a somewhat lesser extent.

Integral equation approaches, using the Green integral representation, are based on the fundamental solution of the leading operator for the governing equation thus being competitive with other well-established methods, such as Finite Difference Methods (FDM), FDTD (suffering from so-called staircasing approximation) or FEM in terms of accuracy and efficiency [5]. Effects of numerical artifacts in low frequency (LF) dosimetry are studied by working group 2 (WG 2) of IEEE/ICES TC95 SC6 EMF Dosimetry Modeling [8].

Applying integral approaches, such as BEM, one avoids; the staircasing error (by use of isoparametric elements), implementation of absorbing boundary conditions and volume meshes for large-scale problems. 
Some disadvantages of integral methods, such as BEM are; more complex fomulation (particularly for non-homogeneous domains) and related numerical implementation (dense matrices, Green function singularities/quasisingularities).

This paper is an extension of recent review papers [5] and [9] and within the framework of WG2 SC6 TC95 tasks gives an overview to the use of some integral methods in electromagnetic dosimetry and biomedical applications of electromagnetic fields. Thus, Laplace equation formulation /BEM scheme for the exposure of human head to electrostatic fields and whole body to LF electric fields is presented. Furthermore, surface integral equation/Moment Method (MoM) approach for the brain exposure is described. This is followed by the tensor volume integral equation/MoM approach applied to the exposure of the whole body to HF radiation. The eye exposure to plane wave is analyzed by using the hybrid BEM/ FEM approach.

\section{INTEGRAL METHODS IN LF DOSIMETRY}

The quasistatic formulation for LF exposures used in this work is based on the Laplace (quasi-static) version of the continuity equation for scalar potential $\varphi$ giveny by [5]:

$$
\nabla \cdot[(\sigma+j \omega \varepsilon) \nabla \varphi]=0
$$

where $\omega$ is the frequency of the incident field, $\sigma$ and $\varepsilon_{r}$ are the conductivity and permittivity of the material.

For the case of human exposure to electrostatic fields (1) simplifies into standard Laplace equation:

$$
\nabla^{2} \varphi=0 .
$$

Knowing the scalar potential along the body, the induced current density is computed from the differential form of Ohm's Law:

$$
\vec{J}=(\sigma+j \omega \varepsilon) \nabla \phi .
$$

The electric field $E$ can be readily obtained as follows:

$$
\vec{E}=-\nabla \phi .
$$

First computational example is related to the human head exposed to video display unit (VDU) at $\varphi s=15 \mathrm{kV}$ at the distance $1 \mathrm{~s}=0.4 \mathrm{~m}$.

The boundary integral representation of (2) is given, as follows [5]:

$$
c_{i} \phi_{i}=\int_{\Gamma} \Psi \frac{\partial \phi}{\partial n} d \Gamma-\int_{\Gamma} \phi \frac{\partial \Psi}{\partial n} d \Gamma
$$

where $\psi$ is the fundamental solution Laplace equation:

$$
\psi=(4 \pi R)^{-1} .
$$

with $R$ being the distance between the source point and observation point in $\Gamma$, and:

$$
c_{i}=\left\{\begin{array}{ll}
1, & i \in \Omega \\
1 / 2, & i \in \Gamma(\text { smooth boundary })
\end{array} .\right.
$$


Fig. 1 shows the electrostatic field induced at the surface of the female face calculated via BEM and FEM, respectively. The details of the head model can be found in [5] and [10]. Comparison of the BEM and FEM results for specific points on the head are shown in Fig. 2.

Comparison of the BEM and FEM results for specific points on the head are shown in Fig. 2.

The details of the FEM solution can be found elsewhere, e.g. in [10]. Differences on the certain points, (nose tip in particular) between the BEM and the FEM solution arise due to difficulties to accomplish sufficiently refined mesh around complex geometries, such as the human face.

Next example is the pregnant woman/foetus exposure to power line electric field $E=10$ $\mathrm{kV} / \mathrm{m}$. The amniotic fluid (AF) has the highest conductivity which varies depending on the period of gestation. Kidney, muscle bone cortical, bladder, spleen, skin have conductivity very close to $0.1 \mathrm{~S} / \mathrm{m}$, while the ovary and cartilage conductivity is around $0.2 \mathrm{~S} / \mathrm{m}$. More details on electrical properties of the pregnant woman tissue can be found elsewhere, e.g. in [11].
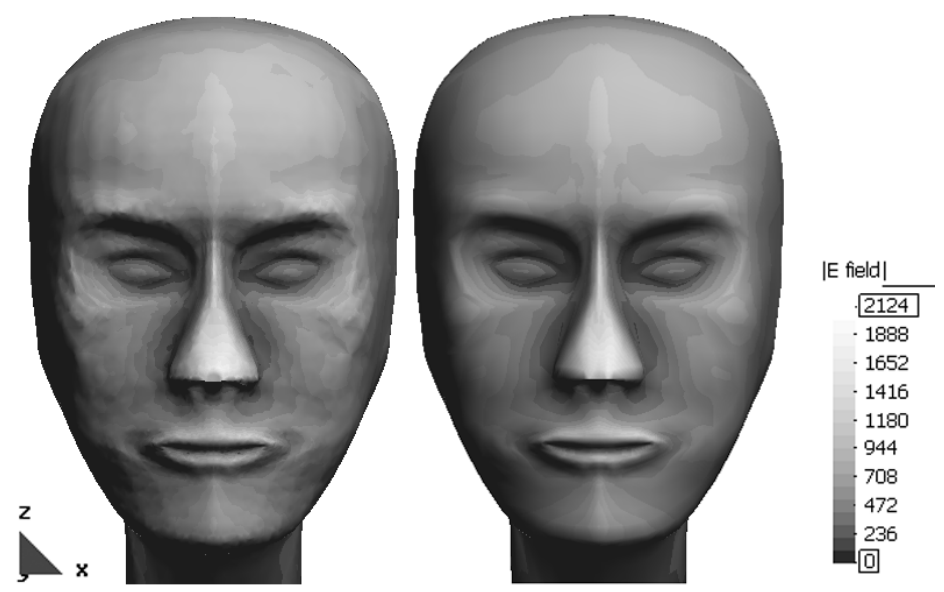

Figure 1: Electrostatic field on the female face. (a) FEM solution; and (b) BEM solution.

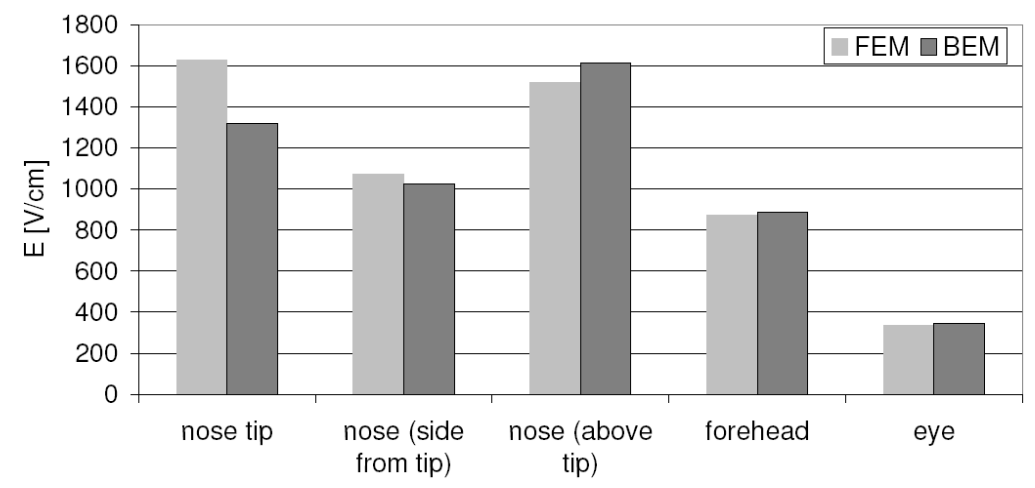

Figure 2: Electrostatic field at the specific locations of the female face. 
Fig. 3 shows the sliced model of the pregnant woman at 26th gestational week with the electric field/scalar potential lines for the foetus in cephalic and breach presentation, respectively.

It is visible that the uterus, due to the higher conductivity of the amniotic fluid compared to the maternal tissue, tends to concentrate the field lines.

The maximal value of current density in the foetus occurs during the 8th week, while the maximum current density induced in the foetus is $7.4 \mathrm{~mA} / \mathrm{m}^{2}$ for an external field exposure of $10 \mathrm{kV} / \mathrm{m}$. Since restriction recommended for public exposure by ICNRP [5] is $2 \mathrm{~mA} / \mathrm{m} 2$. Then through the modelling it is possible to set restrictions on external field exposure, which translate to a maximum external field of $2.7 \mathrm{kV} / \mathrm{m}$ in order to limit the current density exposure in the earlier months of development.

\section{INTEGRAL METHODS IN HF DOSIMETRY AND BIOMEDICAL APPLICATIONS} The main task of HF dosimetry is to quantify thermal effects, by determining the level and distribution of the electromagnetic energy absorbed by the body which is expressed in terms of the specific absorption rate $(S A R)$. Standard definition of $S A R$ is given by the rate of energy $W$ absorbed by the unit body mass $m$ :

$$
S A R=\frac{d P}{d m}=\frac{\sigma}{2 \rho}|E|^{2},
$$

where $P$ is the dissipated power, $E$ is the peak value of the electric field, respectively, $\rho$ is the tissue density and $\sigma$ is the tissue conductivity.
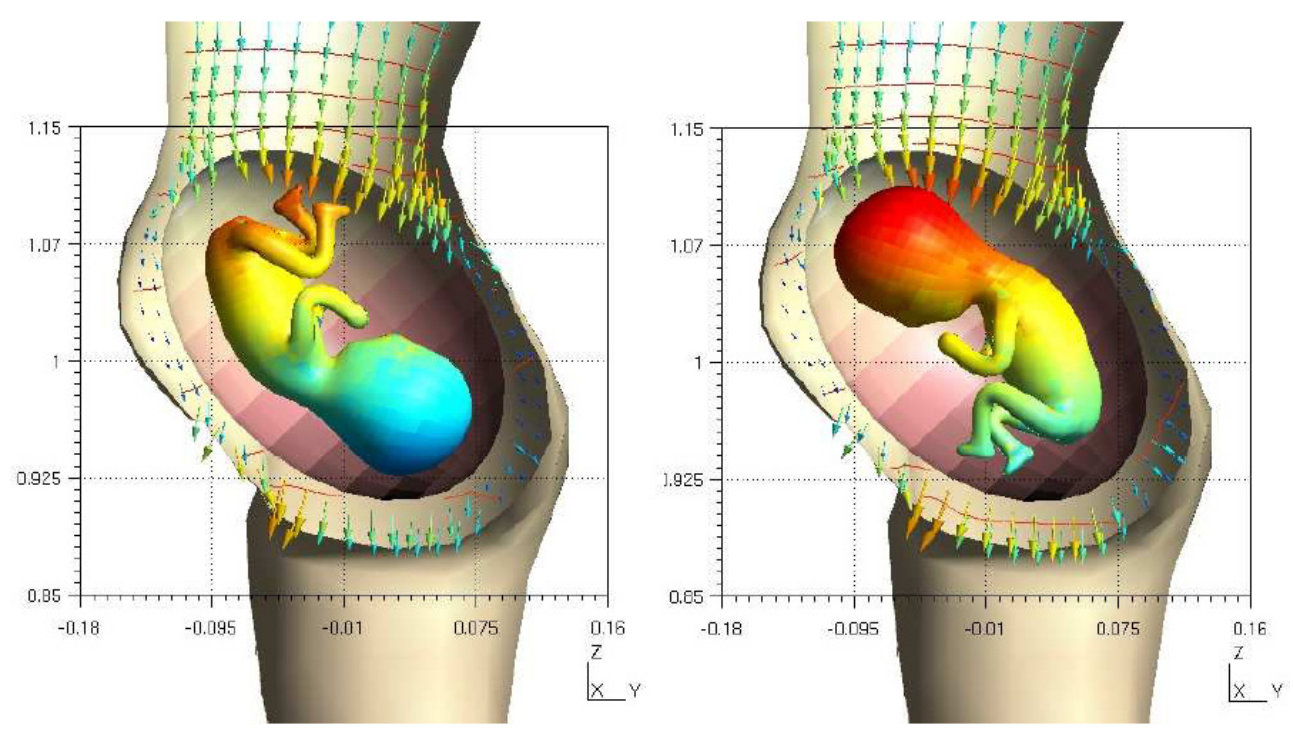

Figure 3: Lateral view of the pregnant woman at 26th gestational week. (a) Foetus in the cephalic presentation; and (b) Foetus in the breach presentation. 
3.1 Set of coupled surface integral equations (SIE) - solution via Method of Moments (MoM)

The brain exposed to HF radiation is analysed by solving the coupled surface integral equations (SIE) [12]:

$$
\begin{aligned}
& j \omega \mu_{n} \iint_{S} \vec{J}\left(\vec{r}^{\prime}\right) G_{n}\left(\vec{r}, \vec{r}^{\prime}\right) \mathrm{d} S^{\prime}- \\
& -\frac{j}{\omega \varepsilon_{n}} \iint_{S} \nabla_{S}^{\prime} \cdot \vec{J}\left(\vec{r}^{\prime}\right) \nabla G_{n}\left(\vec{r}, \vec{r}^{\prime}\right) \mathrm{d} S^{\prime}+ \\
& +\iint_{S} \vec{M}\left(\vec{r}^{\prime}\right) \times \nabla^{\prime} G_{n}\left(\vec{r}, \vec{r}^{\prime}\right) \mathrm{d} S^{\prime}= \begin{cases}\vec{E}^{i n c}, & n=1 \\
0, & n=2,\end{cases}
\end{aligned}
$$

where $\vec{J}$ and $\vec{M}$ is quivalent electric and magnetic current density, respectively, $k_{\mathrm{n}}$ is the wave number of a medium $n$, while $G_{\mathrm{n}}$ is the interior/exterior Green function for the homogeneous medium [12]:

$$
G_{n}\left(\vec{r}, \vec{r}^{\prime}\right)=\frac{e^{-j k_{n} R}}{4 \pi R} ; \quad R=\left|\vec{r}-\vec{r}^{\prime}\right|,
$$

and $R$ is the distance from the source to observation point, respectively while $k$ n denotes the wave number of a medium $n,(n=1 ; 2)$.

Set of integral eqn (9) is solved via the Method of Moments (MoM) procedure presented in [12].

The equivalent electric and magnetic current densities $\vec{J}$ and $\vec{M}$ in (9) are expressed in terms of a linear combination of basis functions $\vec{f}_{n}$ and $\vec{g}_{n}$ :

$$
\begin{gathered}
\vec{J}(\vec{r})=\sum_{n=1}^{N} J_{n} \vec{f}_{n}(\vec{r}), \\
\vec{M}(\vec{r})=\sum_{n=1}^{N} M_{n} \vec{g}_{n}(\vec{r}),
\end{gathered}
$$

where $J_{\mathrm{n}}$ and $M_{\mathrm{n}}$ are unknown coefficients, while $N$ is the total number of triangular elements.

Utilizing the weighted residual approach, i.e. multiplying (9) by the set of a weight functions $\vec{f}_{m}$ and integrating over the surface $S$, after certain mathematical manipulations, it follows: 


$$
\begin{aligned}
& j \omega \mu_{i} \sum_{n=1}^{N} J_{n} \iint_{S} \vec{f}_{m}(\vec{r}) \cdot \iint_{S^{\prime}} \vec{f}_{n}\left(\vec{r}^{\prime}\right) G_{i} d S^{\prime} d S+ \\
& +\frac{j}{\omega \varepsilon_{i}} \sum_{n=1}^{N} J_{n} \iint_{S} \nabla_{S} \cdot \vec{f}_{m}(\vec{r}) \iint_{S^{\prime}} \nabla_{S}^{\prime} \cdot \vec{f}_{n}\left(\vec{r}^{\prime}\right) G_{i} d S^{\prime} d S+ \\
& \pm \sum_{n=1}^{N} M_{n} \iint_{S} \vec{f}_{m}(\vec{r}) \cdot\left[\hat{n} \times \vec{g}_{n}\left(\vec{r}^{\prime}\right)\right] d S+ \\
& +\sum_{n=1}^{N} M_{n} \iint_{S} \vec{f}_{m}(\vec{r}) \cdot \iint_{S^{\prime}} \vec{g}_{n}\left(\vec{r}^{\prime}\right) \times \nabla^{\prime} G_{i} d S^{\prime} d S= \\
& = \begin{cases}\iint_{S} \vec{f}_{m}(\vec{r}) \cdot \vec{E}^{i n c} d S & , i=1 \\
0 & , i=2,\end{cases}
\end{aligned}
$$

where subscript $i$ is the index of the medium. The details of the procedure could be found elsewhere, e.g. in [12].

Fig. 4 shows the $S A R$ distribution in the brain at $f=900 \mathrm{MHz}$ due to the vertically polarized incident plane wave with power density $P=5 \mathrm{~mW} / \mathrm{cm}^{2}$. The brain electrical parameters are: $\varepsilon_{\mathrm{r}}=46, \sigma=0.8 \mathrm{~S} / \mathrm{m}[12]$.

The obtained numerical results for peak and average SAR values are: $S A R_{\max }=0.866 \mathrm{~W} / \mathrm{kg}$ and $S A R_{\text {avg }}=0.158 \mathrm{~W} / \mathrm{kg}$.
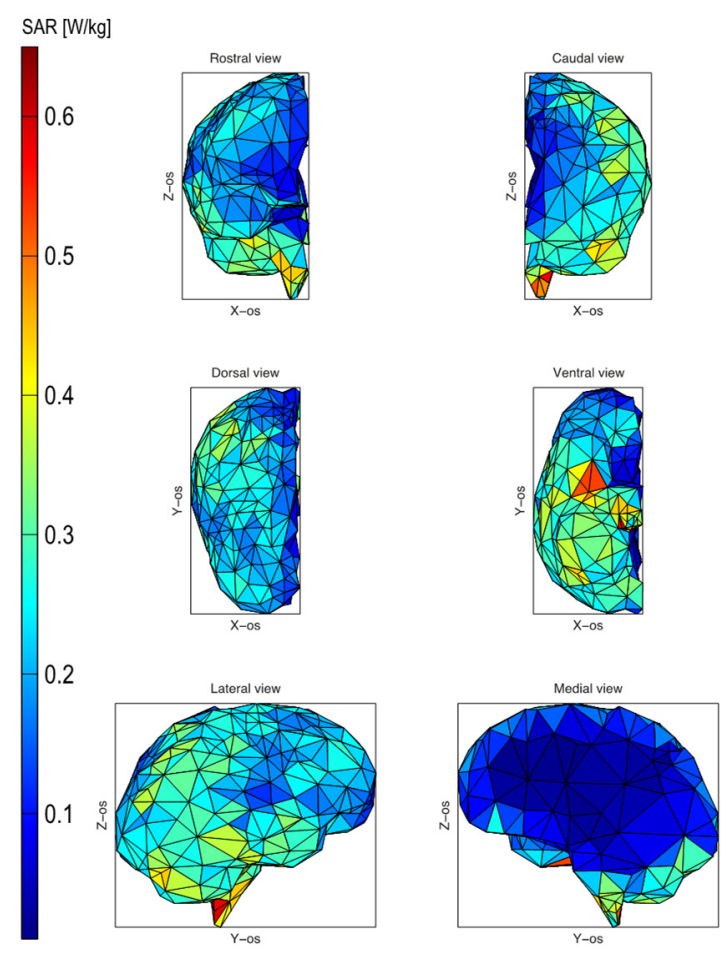

Figure 4: $S A R$ distribution at $f=900 \mathrm{MHz}$. 


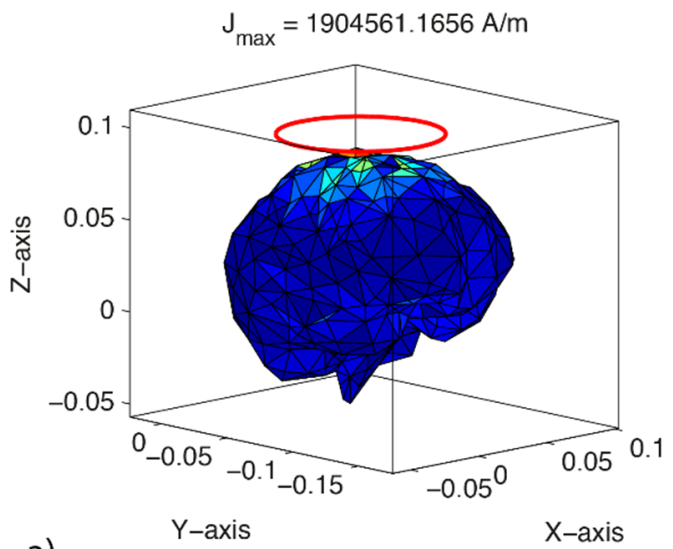

a)

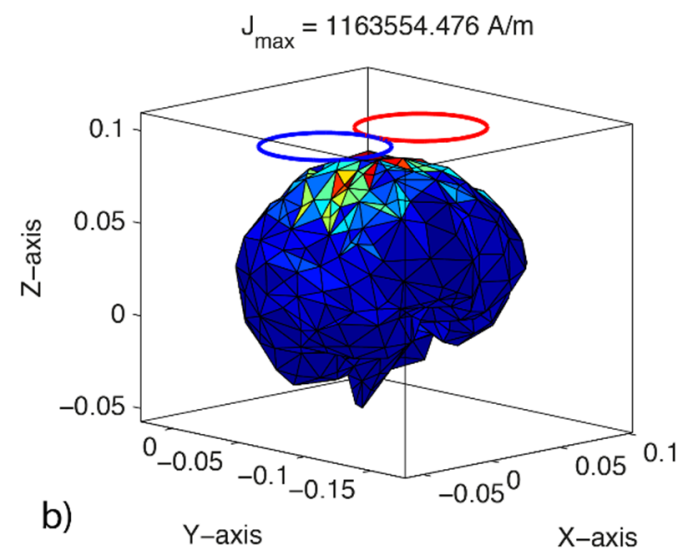

Figure 5: Equivalent electric current density. (a) Circular coil; and =(b) No 8 coil.

Additional example deals with the transcranial magnetic stimulation (TMS) treatment for the case of exposure to circular coil and No 8 coil. The numerical results for the electric current density induced in the brain are presented in Fig. 5.

More details can be found elsewhere, e.g. in [13].

\subsection{Tensor volume integral equation/MoM approach}

The whole-body exposure to HF radiation can be analysed via tensor integral eqn (14). The internal field due to plane wave incident field $E^{\mathrm{i}}$ is governed by the tensor volume integral eqn (14):

$$
\left[1+\frac{\tau(\vec{r})}{3 j \varpi \varepsilon_{0}}\right] \vec{E}(\vec{r})-P V \int_{v} \tau(\vec{r}) \vec{E}(\vec{r}) \cdot \underline{G}\left(\vec{r}, \vec{r}^{\prime}\right) d v^{\prime}=\vec{E}^{i}(\vec{r}),
$$

where PV is the principal value and $\tau(\vec{r})=\sigma+j \varpi\left[\varepsilon(\vec{r})-\varepsilon_{0}\right]$. 
Integral eqn (14) is solved via (MoM) by discretising the body into $N$ subvolumes $V_{\mathrm{m}}$ with $E$ and $\tau$ assumed to be constant within each cell. If $\vec{r}_{m}$ is the center of the $m$ th cell, it follows:

$$
\left[1+\frac{\tau(\vec{r})}{3 j \varpi \varepsilon_{0}}\right] E_{x_{p}}\left(\mathbf{r}_{m}\right)-\sum_{q=1}^{3}\left[\sum_{q=1}^{3} \tau(\vec{r}) P V \int_{V_{m}} G_{x_{p} x_{q}}\left(\vec{r}_{m}, \vec{r}^{\prime}\right) d v^{\prime}\right] E_{x_{q}}\left(\vec{r}_{n}\right)=E_{x_{p}}\left(\vec{r}_{m}\right),
$$

where $G_{x_{p} x_{q}}$ is the tensor Green function given by:

$$
G_{x_{p} x_{q}}\left(\vec{r}, \vec{r}^{\prime}\right)=-j \varpi \mu_{0}\left[\delta_{p q}+\frac{1}{k_{0}^{2}} \frac{\partial^{2}}{\partial x_{p} \partial x_{q}}\right] G_{0}\left(\vec{r}, \vec{r}^{\prime}\right), \mathrm{p}, \mathrm{q}=1,2,3,
$$

where $m, n=1,2, \ldots, N, p, q=1,2,3$.

Numerical solution details can be found elsewhere, e.g. in [14]. The computational example is related to the whole-body exposure to UMTS base station antenna system at $f=2.14 \mathrm{GHz}$ mounted on roof at the height $h=15 \mathrm{~m}$. The human being is located approximately $24 \mathrm{~m}$ from the base station. The measured value of the electric field illuminating the body is $14.87 \mathrm{~V} / \mathrm{m}$. The human body parameters are: $\varepsilon_{\mathrm{r}}=53.11$ and $\sigma=1.54 \mathrm{~S} / \mathrm{m}$, i.e. it follows: $\tau=$ $1.54+j 6.1978$ [9]. The maximum value of SAR induced in the body is $0.31 \mathrm{~mW} / \mathrm{kg}$, in the region of the waist. The computed value of the whole-body average SAR is $82.4701 \mu \mathrm{W} / \mathrm{kg}$ which stay far below the ICNIRP exposure limit.

\subsection{Straton Chu formulation/hybrid BEM/FEM solution}

Plane wave incident on the corneal part of the eye, treated as an exterior unbounded scattering problem is formulated via the Stratton-Chu formulation, i.e. the time-harmonic electric field at the exterior domain is expressed by the following boundary integral eqn (5):

$$
\alpha \vec{E}=\vec{E}_{i}+\oint_{\partial V^{\prime}} \vec{n}^{\prime} \times\left(\nabla^{\prime} \times \vec{E}\right) G d S^{\prime}+\oint_{\partial V^{\prime}}\left[\left(\vec{n}^{\prime} \times \vec{E}\right) \times \nabla^{\prime} G+\left(\vec{n}^{\prime} \cdot \vec{E}\right) G\right] d S^{\prime},
$$

where $E_{\mathrm{i}}$ is the incident electric field.

The interior domain is governed by the vector Helmholtz equation [5]:

$$
\nabla \times\left(\frac{1}{k_{B}} \nabla \times \vec{E}\right)-k_{A} \vec{E}=0
$$

where subscripts $\mathrm{A}$ and $\mathrm{B}$ denote the exterior and interior region, respectively.

This coupled formulation (17), (18) is treated via hybrid BEM/FEM technique. The eye parameters and mathematical details are available elsewhere, e.g. in [5].

Fig. 6 shows the $S A R$ induced in the exposed to plane wave with power density of 10 $\mathrm{W} / \mathrm{m}^{2}$.

The whole eye averaged $S A R$ at $f=1 \mathrm{GHz}$ is $0.3352 \mathrm{~W} / \mathrm{kg}$ being below ICNIRP limits [2]. 


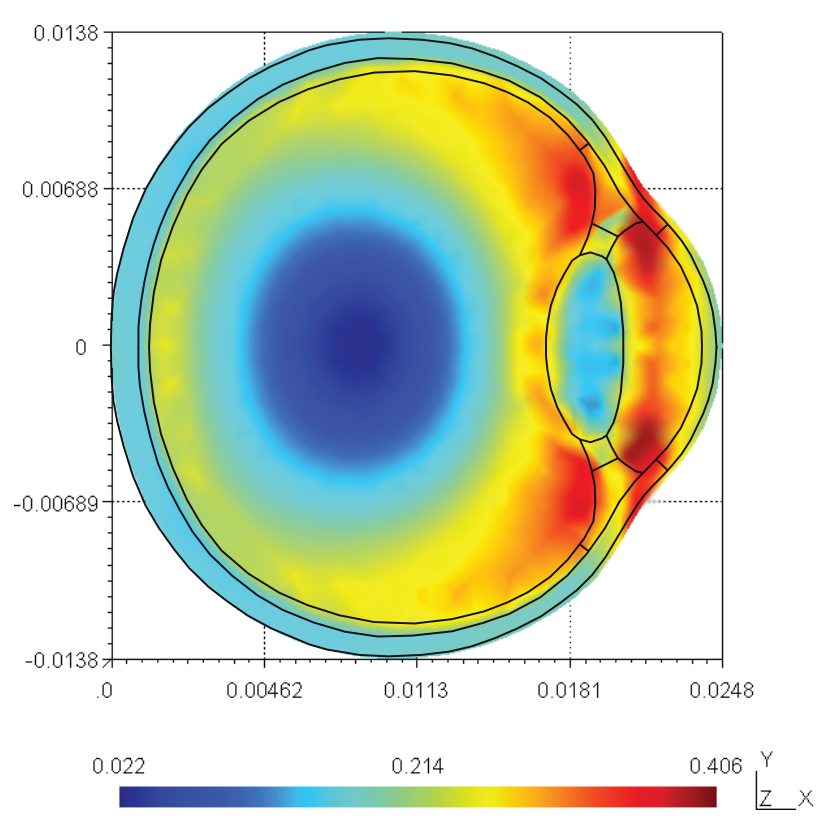

Figure 6: SAR in the eye due to plane wave exposure $10 \mathrm{~W} / \mathrm{m}^{2}$ at $f=1 \mathrm{GHz}$.

\section{CONCLUDING REMARKS}

An overview of the use of certain boundary integral methods of solution in bioelectromagnetics and biomedical applications of electromagnetic fields is given in the paper. A trade-off between integral boundary and differential domain methods, respectively, is given, as well.

Thus, static and LF dosimetry techniques based on the Laplace equation /BEM solution are analysed. Furthermore, HF dosimetry methods are analysed; Surface Integral Equation/Metod of Moments (MoM), tensor volume integral equation/MoM and StrattonChu integral formulation/Helmholtz equation treated with hybrid boundary element method/finite element method (BEM/FEM) scheme. Some illustrative computational examples are also presented.

\section{REFERENCES}

[1] Poljak, D., Electromagnetic fields: Environmental exposure. Encyclopedia of Environmental Health, ed. J.O. Nriagu, Elsevier: Burlington, 2, pp. 259-268, 2011.

[2] International Commission on Non-Ionizing Radiation Protection, "Guidelines for limiting exposure to time-varying electric, magnetic and electromagnetic fields (up to 300 GHZ)”. Health Physics, 74(4), pp. 494-522, Apr. 1998.

[3] International Commission on Non-Ionizing Radiation Protection, "Guidelines for limiting exposure to time-varying electric and magnetic fields (1 HZ-100 kHZ)". Health Physics, 99(6), pp. 818-836; Dec. 2010.

[4] Hand, J.W., Modeling the interaction of electromagnetic fields (10 MHz-10 GHz) with the human body: methods and applications. Physics in Medicine and Biology, 53(16), pp. 243-286, 2008. DOI: 10.1088/0031-9155/53/16/r01. 
[5] Poljak, D., Cavka, D., Dodig, H., Peratta, C. \& Peratta, A., On the use of boundary element analysis in bioelectromagnetics. Engineering Analysis with Boundary Elements, 49, pp. 2-14, 2014. DOI: 10.1016/j.enganabound.2014.02.008.

[6] Singh, K.D., Longan, N.S. \& Gilmartin, B., Three dimensional modeling of the human eye based on magnetic resonance imaging. Investigative Opthamology \& Visual Science, 47(6), pp. 2272-2279, 2006. DOI: 10.1167/iovs.05-0856.

[7] Hirata, A., Temperature increase in human eyes due to near-field and far-field exposures at $900 \mathrm{MHz}, 1.5 \mathrm{GHz}$, and $1.9 \mathrm{GHz}$. IEEE Transactions on Electromagnetic Compatibility, 47(1), pp. 68-76, 2005. DOI: 10.1109/temc.2004.842113.

[8] Reilly, J.P. \& Hirata, A., Low-frequency electrical dosimetry: Research agenda of the IEEE international committee on electromagnetic safety. Physics in Medicine and Biology, 61(12), R138-R149, 2016. DOI: 10.1088/0031-9155/61/12/r138.

[9] Poljak, D., Cvetković, M., Peratta, A., Peratta, C., Dodig, H. \& Hirata, A., On Some Integral Approaches in Electromagnetic Dosimetry, The Joint Annual Meeting of The Bioelectromagnetics Society and the European BioElectromagnetics Association. BioEM: Gent, 289-295, 2016.

[10] Čavka, D., Poljak, D. \& Peratta, A., Comparison between finite and boundary element methods for analysis of electrostatic field around human head generated by video display units. Journal of Communications Software and Systems, 7(1), pp. 22-28, 2011. DOI: $10.24138 /$ jcomss.v7i1.184.

[11] Poljak, D., On the assessment of pregnant woman/foetus exposure to electromagnetic fields by using numerical methods. Paediatria Croatica, 57(Supl 1), pp. 1-9, 2013.

[12] Cvetković, M. \& Poljak, D., An efficient integral equation based dosimetry model of the human brain. International Symposium on Electromagnetic Compatibility (EMC Europe 2014), Gothenburg, Sweden, pp. 375-380, Sep. 1-4, 2014.

[13] Poljak, D. et al., Integral equation models in some biomedical applications of electromagnetic fields: Transcranial magnetic stimulation (TMS), nerve fiber stimulation. International Multidisciplinary Conference on Computer and Energy Science (SpliTech), pp. 1-6, 2016. DOI: 10.1109/splitech.2016.7555936.

[14] Sadiku, M.N.O., Numerical Techniques in Electromagnetics, 2nd ed., CRC Press: New York, 2001. 